\title{
Consumption of Sugar-Sweetened Beverages in Relation to the Metabolic Syndrome among Iranian Adults
}

\author{
Hossein Khosravi-Boroujeni $^{a} \quad$ Nizal Sarrafzadegan ${ }^{b}$ \\ Noushin Mohammadifard ${ }^{\mathrm{b}}$ Hasan Alikhasi $^{\mathrm{b}}$ Firouzeh Sajjadi $^{\mathrm{b}}$ \\ Sedigheh Asgari ${ }^{b}$ Ahmad Esmaillzadeh ${ }^{c}$ \\ ${ }^{a}$ Cardiac Rehabilitation Research Center, ${ }^{b}$ Isfahan Cardiovascular Research Center, \\ Isfahan Cardiovascular Research Institute, ' Food Security Research Center, Department of \\ Community Nutrition, School of Nutrition and Food Science, Isfahan University of Medical \\ Sciences, Isfahan, Iran
}

\section{Key Words}

Sugar-sweetened beverages $\cdot$ Metabolic syndrome $\cdot$ Food frequency questionnaire $\cdot$ Diet

\begin{abstract}
Background: Few data are available linking intake of sugar-sweetened beverages (SSBs) with the metabolic syndrome. Furthermore, findings from these studies are inconsistent and most are from Western societies; no information is available in this regard from Middle Eastern populations. Objective: This study was conducted to assess the relationship between SSB consumption and metabolic syndrome in an Iranian adult population. Methods: In this crosssectional study, data from 1,752 people (782 men and 970 women) that were selected with the multistage cluster random sampling method from three counties of Isfahan, Najafabad and Arak were used. A validated food frequency questionnaire was used to assess participants' usual dietary intakes. Consumption of SSBs was calculated by summing up the consumption of 'soft drinks' and 'artificially sweetened fruit juices'. To categorize participants, we used three levels of SSB consumption: $<1$ time/week, 1-3 times/week, and $>3$ times/week. Biochemical assessments were done after an overnight fasting. Metabolic syndrome was defined according to the guidelines of Adult Treatment Panel III. Results: Subjects with high consumption of SSBs ( $>3$ times/week) were younger than those with low consumption $(<1$ time/week). Mean BMI was not significantly different across SSB categories. High consumption of SSBs was associated with greater intakes of energy and almost all food groups. We found a significant difference in serum triglyceride levels between men consuming SSBs 1-3
\end{abstract}


times/week and those consuming $<1$ time/week. However, after controlling for potential confounders, this association disappeared. In crude models, no significant associations were found between SSB intake and prevalence of the metabolic syndrome in either gender. After adjustment for potential confounders including BMI, we found that men in the top category of SSB intake were $17 \%$ more likely to have the metabolic syndrome (odds ratio (OR) 1.17; 95\% confidence interval ( $95 \%$ CI) $0.56-2.46)$, while women in the highest category were $20 \%$ less likely to have the syndrome (OR $0.80 ; 95 \%$ CI 0.46-1.42) as compared with those in the bottom category. However, these associations were not significant in either men or women. Conclusion: Our results do not support the previous findings on the association between SSB consumption and metabolic syndrome. Prospective studies are needed to further explore for this association.

Copyright (c) 2012 S. Karger GmbH, Freiburg

\section{Introduction}

The metabolic syndrome is a conglomerate of metabolic abnormalities that raise individual's risk for type 2 diabetes mellitus [1], cardiovascular disease [2], and other chronic diseases [3]. It has been defined to include abdominal obesity, high blood pressure, elevated fasting plasma glucose (FPG) and triglycerides, and low high-density lipoprotein (HDL) cholesterol [4]. Metabolic syndrome is associated with non-alcoholic hepatitis and polycystic ovary disease [5]. A role for metabolic syndrome in the development of some cancers has also been suggested [5]. Due to increasing prevalence of obesity worldwide, the number of individuals with metabolic syndrome is continuing to rise. One out of four Americans is affected by the metabolic syndrome [6]. Recent estimates in Iran have shown that the syndrome is highly prevalent among Iranians such that more than $30 \%$ of adults [7] and almost $10 \%$ of adolescents [8] are affected.

The metabolic syndrome is strongly related to lifestyle factors including diet $[9,10]$, smoking [11], and physical activity [12]. Dietary determinants of the metabolic syndrome have been summarized in a recent review [13], indicating that high intakes of fruit and vegetables [9], dairy products [14] and whole grains [15] are associated with lower risk while increased consumption of refined carbohydrates and red meat is associated with greater risk of the syndrome $[14,16]$.

Recently, consumption of sugar-sweetened beverages (SSBs) has greatly increased in the USA, Europe [17, 18], and even in developing countries $[19,20]$. Consumption of soft drinks has been related to the prevalence of obesity and diabetes in children and adolescents and also to the development of hypertension in adults [21,22]. Added sugars in soft drinks have also been associated with elevated levels of serum triglycerides and insulin resistance [23]. This might be explained by greater caloric intake, less satiety, and greater insulin secretion [24]. Therefore, consumption of soft drinks can cause metabolic dysfunction that lead to the metabolic syndrome. Few data are available linking SSB intake and metabolic syndrome. Furthermore, findings from these studies are inconsistent. Some crosssectional [14,25] and prospective studies [26] have demonstrated that intake of 'regular' and 'diet drinks' have been positively related to metabolic syndrome, while a recent prospective study failed to reach a significant association between SSB intake and incidence of metabolic syndrome [13]. All these studies came from Western societies; no information is available in this regard from Middle Eastern populations. Finding associations between SSB intake and metabolic syndrome is particularly relevant for this region because consumption of SSBs in this part of the world has increased tremendously due to the phenomenon of nutrition transition $[19,20]$. In addition, prevalence of the metabolic 
syndrome among Iranian women has been reported to be among the highest rates in the world $[8,27]$. Therefore, examining the association between SSB consumption and the metabolic syndrome in this part of the world might add further information to the knowledge in this field. This study aimed to evaluate the association between consumption of SSBs and metabolic syndrome in a large group of the Iranian population.

\section{Participants and Methods}

\section{Participants}

This cross-sectional study was based on the first part of Isfahan Healthy Heart Program (IHHP). The IHHP was a community-based program for prevention and control of cardiovascular disease and healthy lifestyle promotion. IHHP started in 1999 and took 7 years until $2006[20,28]$. This program was conducted by the Isfahan Cardiovascular Research Institute and the Isfahan Provincial Health Office. From three provincial cities of Isfahan, Najafabad and Arak, more than 12,000 men and women older than 19 years were selected. For sampling process which has been described elsewhere in more detail [28, 29], multistage cluster random sampling method was used. In the current study, participants of IHHP who had complete data on dietary intakes, anthropometric measures, FPG, and serum lipid profiles as well as data for confounding variables were included. After excluding individuals with a prior history of chronic diseases, those who reported a total energy intake of $<800$ or $>4,200 \mathrm{kcal}$, and those taking medications, a total of 1,752 people (782 men and 970 women) remained for the current analysis. Each participant provided a signed written informed consent. The study was approved by the Ethics Committee of Isfahan University of Medical Sciences and other relevant national organizations.

\section{Assessment of Dietary Intake}

Trained technicians assessed dietary intakes of participants by the use of a 49-item food frequency questionnaire (FFQ). The validity of this questionnaire was confirmed by Medical Education Development Center before being used [28]. Participants were asked to report frequency of consumption for each food item during the last year. These data were collected based on daily (e.g. bread), weekly (e.g. rice, meat), and monthly (e.g. fish) basis. For the present analysis, all data on food items were computed as daily intakes by dividing weekly consumption by 7 and monthly consumption by 30. Consumption of SSBs was calculated by summing up the consumption of 'soft drinks' and 'artificially sweetened fruit juices' (commonly used in Iran). Individuals with 3 or more times/week consumption of SSBs were defined as high consumers. Such definition is different from those reported from Western countries. This discrepancy in definition can be explained by lower consumption of SSBs in Iran compared with Western populations.

\section{Biochemical Assessment}

All biochemical measurements were done on a fasting blood sample taken from each participant after an overnight fast. FPG was measured on the day of blood collection by enzymatic colorimetric method using glucose oxidase (Pars Azmoon Inc., Tehran, Iran). For further biochemical analyses, the collected blood samples in each center were frozen at $-20^{\circ} \mathrm{C}$ until being assayed in the central laboratory of Isfahan Cardiovascular Research Center, a WHO collaborating center. Enzymatic colorimetric method was used to measure serum total cholesterol and triglyceride levels. Dextran sulphate-magnesium chloride was used to remove non-HDL cholesterol. After precipitation of the apolipoprotein B-containing lipoproteins, HDL cholesterol levels were determined by the use of commercially available enzymatic kits. To quantify serum low-density lipoprotein (LDL) cholesterol levels, we used the Friedewald equation [30]. The metabolic syndrome was defined based on Adult Treatment Panel III, such that individuals with 3 or more of the following risk factors were described as having metabolic syndrome: FPG of $>100 \mathrm{mg} / \mathrm{dl}$, waist circumference of $\geq 102 \mathrm{~cm}$ for men and $\geq 88 \mathrm{~cm}$ for women, serum triglyceride levels of $\geq 150 \mathrm{mg} / \mathrm{dl}$, serum HDL cholesterol levels of $<40 \mathrm{mg} / \mathrm{dl}$ for men and $<50 \mathrm{mg} / \mathrm{dl}$ for women, and systolic or diastolic blood pressure of $\geq 130 / 85 \mathrm{~mm} \mathrm{Hg}$.

\section{Assessment of Other Variables}

Trained interviewers collected information about socioeconomic and demographic data in a face-toface method. Data on medical and family history as well as smoking status were collected by the use of 

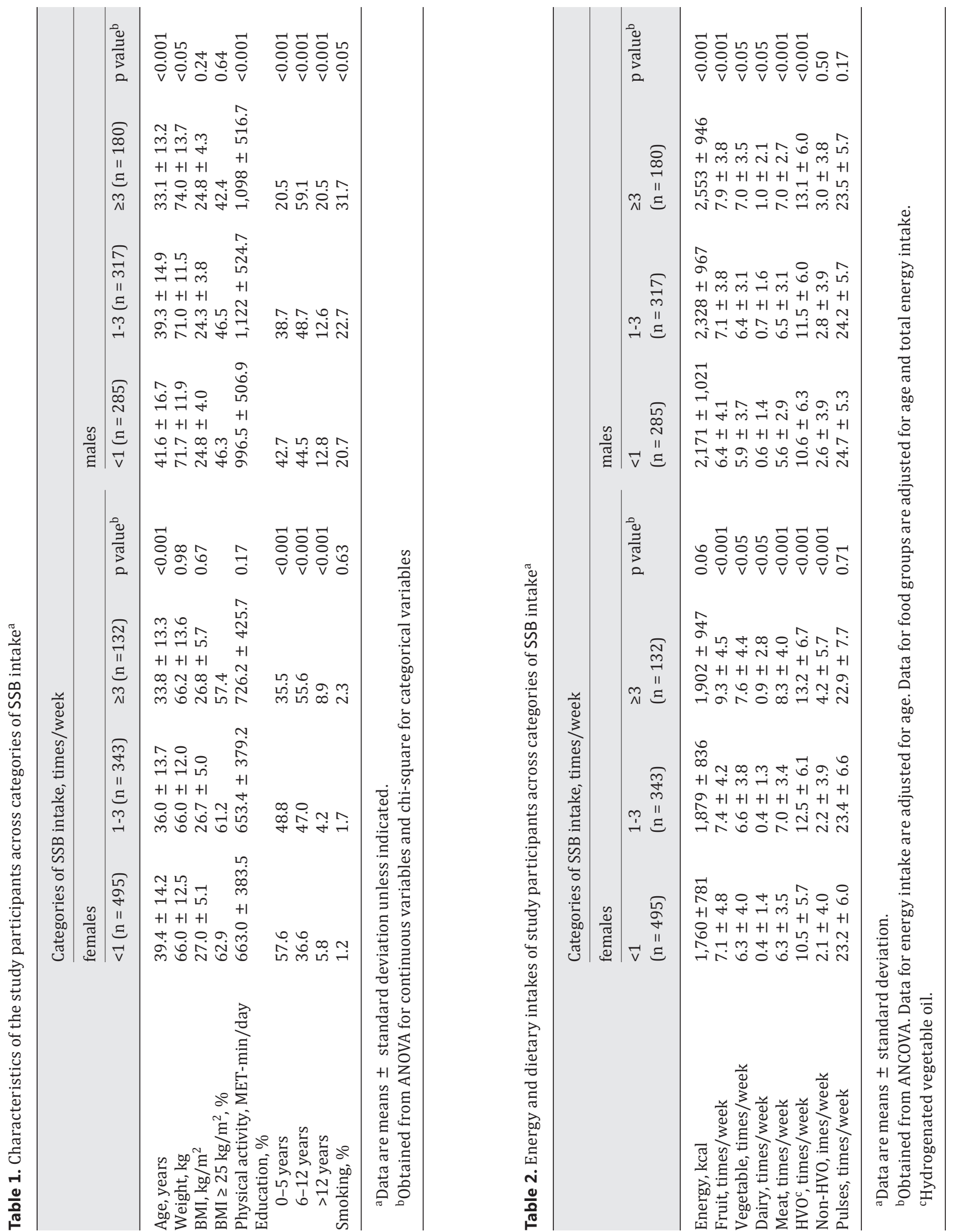
pretested questionnaires. Physical activity levels of participants were assessed using the Baecke questionnaire [31]. A secured metal ruler was used to measure height in barefoot. A calibrated scale was used to measure weight in light clothing. BMI was calculated as weight in kilogram divided by height in meters squared. Waist circumference was taken as the smallest circumference around costal margin and hip circumference was taken at the greatest circumference. Blood pressure was measured twice in a seated position after a 5-min rest. The average of two measurements was considered as the final systolic and diastolic blood pressure.

\section{Statistical Methods}

Data analysis was done separately by sex. To categorize participants, we used three levels of SSB consumption: $<1$ time/week, 1-3 times/week and >3 times/week. General characteristics of the study participants across categories of SSB intake were assessed by the use of analysis of variance (for continuous variables) and chi-square tests (for categorical variables). We used analysis of covariance for comparing dietary intakes across categories of SSB consumption with age and total energy intake as covariates. Multivariate-adjusted means of metabolic risk factors across categories of SSB intake were obtained by the use of analysis of covariance with age (continuous), daily physical activity (continuous), smoking status (current, non-current), total energy intake (kcal/day), and consumption of meats, fruits, vegetables, pulses, dairy, hydrogenated and non-hydrogenated vegetable oils (all as continuous) as covariates. To determine the association of SSB intake with the metabolic syndrome, we used logistic regression analysis. Besides a crude model in this analysis, we constructed three other models with adjustment for age and total energy intake in the first model. We further controlled for daily physical activity (continuous), smoking status (current, non-current), and consumption of meats, grains, fruits, vegetables, pulses, dairy, hydrogenated and non-hydrogenated vegetable oils (all as continuous) in the second model. Finally, additional adjustment for BMI was done to explore if the associations are mediated through obesity. The logistic regressions were first constructed for the whole population; then the stratified analysis was performed according to BMI (BMI $<25$ and BMI $\geq 25 \mathrm{~kg} / \mathrm{m}^{2}$ ) to determine the discrepancy of associations among normal-weight (BMI $<25 \mathrm{~kg} / \mathrm{m}^{2}$ ) and overweight (BMI $\geq 25 \mathrm{~kg} / \mathrm{m}^{2}$ ) participants. In all logistic regression models, the first category of SSB intake ( $<1$ time/week) was considered as a reference. MantelHaenszel extension chi-square test was used to examine the overall trend of odds ratios across categories of SSB intake. Statistical Package for Social Science (SPSS Inc., Chicago IL, USA; Version 16.0) was used for all statistical analyses.

\section{Results}

The characteristics of the study participants across categories of SSB consumption are shown in table 1. Males and females with high consumption of SSBs ( $>3$ times/week) were younger than those with low consumption $(<1$ time/week) ( $p<0.001)$. Mean BMI was not significantly different across SSB categories; however, high SSB consumption was associated with higher weight in men $(\mathrm{p}<0.05)$, but not in women. Males in the highest category of SSB intake were more likely to be current smokers and physically active $(\mathrm{p}<0.05)$. The distribution of individuals in terms of educational level was significantly different across categories of SSB intake $(\mathrm{p}<0.001)$. Prevalence of overweight and obesity was not associated with SSB consumption in either gender.

Dietary intakes of study participants across categories of SSB intake are provided in table 2. High consumption of SSBs was associated with greater intakes of energy and almost all food groups. Women in the highest category of SSB intake consumed higher amounts of fruits, vegetables, dairy, meat as well as hydrogenated and non-hydrogenated vegetable oils as compared with those in the lowest category. This was also the case for total energy intake; however, the association was marginally significant $(\mathrm{p}=0.06)$. Except for consumption of pulses and non-hydrogenated vegetable oils, men in the top category of SSB intake had higher intakes of energy and all food groups as compared with those in the bottom category $(\mathrm{p}<0.001)$. 


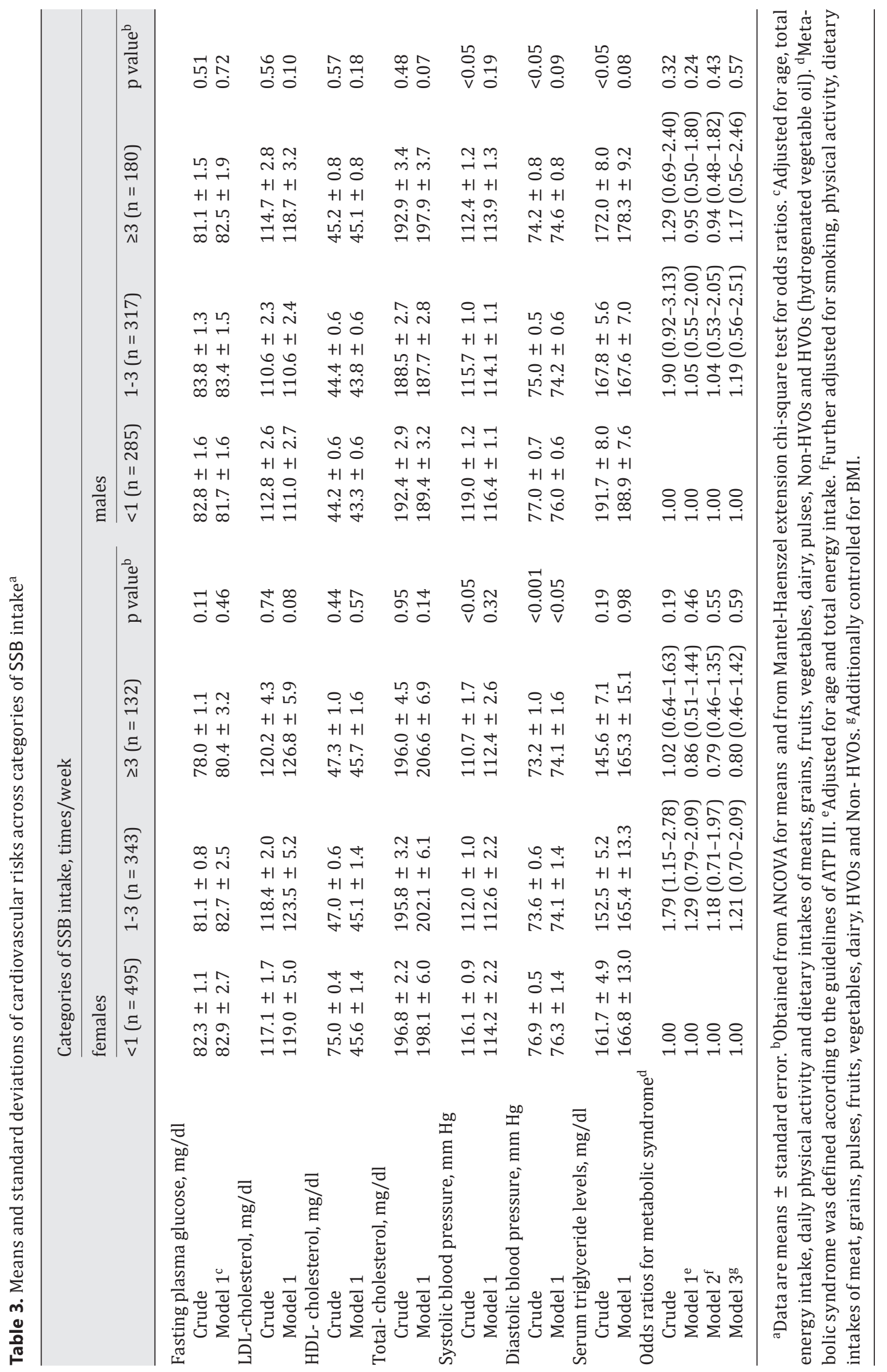


Crude and multivariate-adjusted means of metabolic risk factors across SSB categories are indicated in table 3. Surprisingly, in crude models we reached significant inverse associations between SSB intake and blood pressure in either gender, such that those in the highest category of SSB intake had lower systolic and diastolic blood pressure as compared with those in the lowest category. However, means of both systolic and diastolic blood pressure in all categories of SSB intake were in the normal range. When the analyses were controlled for potential confounders, the associations disappeared, except for the association of SSB intake with diastolic blood pressure among females. Besides blood pressure, we also found a significant difference in serum triglyceride levels between men consuming SSBs 1-3 times/week and those consuming $<1$ time/week. However, after controlling for potential confounders, this association disappeared. No overall significant differences were found in other metabolic risk factors across categories of SSB intake. Multivariate-adjusted odds ratios indicated no significant associations between SSB intake and prevalence of the metabolic syndrome in either gender (table 3). Adjustment for age and total energy intake slightly altered the odds ratios, but the associations were still nonsignificant. Additional control for dietary intakes did not affect the associations in either gender. After further adjustment for BMI, we found that men in the top category of SSB intake were $17 \%$ more likely to have the metabolic syndrome, while women in the highest category were $20 \%$ less likely to have the syndrome as compared with those in the bottom category. However, these associations were not significant in either men or women. No increasing or decreasing trend in the odds ratios of metabolic syndrome were found with increased consumption of SSBs. When a stratified analysis was conducted according to the BMI, the same findings were obtained. This was also the case when the analyses were performed separately by gender (data not shown).

\section{Discussion}

In this cross-sectional study on an Iranian adult population, we failed to find any significant association between SSB intake and metabolic syndrome. Lack of significant associations was persisted even after controlling for potential confounders.

Several potential reasons might explain these findings. The FFQ we used for dietary data collection had no information on portion size, and we had collected data about consumption frequency. Therefore, lack of accuracy on the assessment of SSB intake is a concern in this study. Furthermore, we had not validated our measurements of blood pressure and biochemical indicators. Lifestyle characteristics of the study population with low levels of SSB consumption might also provide additional reasons.

SSBs are carbonated or noncarbonated beverages that contain high amounts of sugar and flavored with natural or artificial additives [32]. Due to the provision of just calorie to the diet, SSBs are usually considered as caloric beverages with harmful effects to human health [33,34]. These kinds of beverages are full of calories and in general are consumed as additional food intake [35]. Recently, SSB consumption has been associated with increased risk of obesity and the metabolic syndrome. However, findings about these associations are inconsistent. In the current study we did not find any significant association between SSB consumption and metabolic syndrome. It must be kept in mind that we defined SSB intake as the sum of 'soft drinks' and 'artificially sweetened fruit juices'. Artificially sweetened fruit juices in Iran are both natural and industrial fruit juices with added sugars. There might be other sugar-containing beverages not considered in our analysis. However, other sugar-containing products such as beverages made with powdered flavoring, non-juice fruit-flavored drinks, sports drinks or sweetened coffee drinks are not of usual intake 
among Iranian general population. These findings are in line with other studies. In a welldesigned prospective study Lutsey et al. [13] showed that consumption of SSBs was not associated with incident metabolic syndrome among middle-aged adults. Findings from a meta-analysis have also indicated that the association between SSB intake and BMI is near zero [36]. Recent reviews have also reached to the conclusion that there is no strong evidence for this association [37-39]. Lack of significant association between SSB consumption and weight gain has also been reported by others [21, 40,41]. Some other studies also reported that there is no convincing evidence to link SSB consumption and obesity [42-45] and diabetes risk [46]. In contrast, others found that high intake of SSBs is positively associated with metabolic syndrome [25, 47, 48], obesity [49-51], related cardio metabolic diseases [52], insulin resistance [42], type 2 diabetes [35, 53, 54], low levels of HDL cholesterol [55], and increased serum triglyceride levels [56]. Consumption of more than one soft drink per day, as compared with less than one drink a day, has been linked with the high prevalence of metabolic syndrome [35]. This has also been shown by a cross-sectional study among young adults [17]. SSB intake in our study is much lower than one serving per day. The SSB intake in the highest category is $>3$ times per week, which is not necessarily the same as once per day. This might explain, to some extent, the discrepancy in findings between this study and earlier ones. Inconsistent findings in different studies might also be explained by different study designs, sample size, or lack of control for confounding variables in some studies [49]. Other factors like different range of BMI studied in different publications as well as the age group of participants might provide some additional reasons for conflicting results [35].

Several mechanisms have been suggested through which SSB consumption might affect risk of the metabolic syndrome. Less satiety-inducing effects of beverages than those of solid foods has been documented as a possible mechanism. Therefore, consumption of energy-containing beverages like SSBs would result in overconsumption [40]. The other mechanism is related to the glycemic effects of the SSBs. SSBs are a high-glycemic-index foods that contain greater amounts of carbohydrates that could rapidly be absorbed and lead to increments in postprandial glucose levels [57]. The association between glycemic index and metabolic syndrome has long been demonstrated [58]. It seems that components other than sugar content of SSBs (for instance additives) might play a role in this association because even the artificially sweetened beverages have been linked with incident metabolic syndrome in the Framingham Heart Study [25] and the Atherosclerosis Risk in Communities Study [13].

Several limitations should be considered in the interpretation of our findings:

First is the cross-sectional design of the study that prohibits inferring causal relationship between SSB intake and the metabolic syndrome. Future longitudinal studies are required to confirm the association. However, it must be kept in mind that the current study comes from a developing country where only limited sources of funding are available for conducting research. Cohort studies are too expensive to be afforded by a developing country. Furthermore, although the association between SSB intake and metabolic syndrome has been reported from Western countries, limited data are available from non-Western populations, particularly from the understudied region of Middle East. In addition, appropriate analysis of cross-sectional data represents a valuable initial step in identifying relationships between diet and disease. Moreover, prospective cohort studies and clinical trials have their own weaknesses.

Second, as we used a FFQ for assessing dietary intakes, misclassification is a major concern in our study as in any other epidemiologic studies. This is of particular importance given that we considered the frequency of SSB consumption instead of its quantity. The FFQ we used for dietary data collection had no information on portion size, and we had collected 
data about consumption frequency. However, it must be kept in mind that putting information on portion size in the FFQ does not add so much, particularly in the case of foods that come in natural units (like SSBs). In addition most of the variations in dietary intake of foods have been shown to be explained by frequency of use rather than their portion size, therefore, data on portion size are relatively unimportant. Furthermore, portion sizes are positively correlated with frequency of use meaning that some of the information on portion size is already accounted for by frequency of use. The potential contribution of additional questions on portion size in the FFQ deserves further investigation; however, available data suggest that such questions do not add substantially to the assessment of dietary intake.

Third, some lifestyle factors that might confound the association between SSB intake and the metabolic syndrome may not have been accurately captured and controlled in our analysis, resulting in residual confounding.

Fourth, the risk estimates obtained in our study were not adjusted for all dietary intakes. However, the associations we observed are unlikely to be confounded significantly by other dietary variables because the extensive adjustments we made had minimal impact on the odds ratios.

Fifth, we excluded individuals taking medications that would affect serum lipid profiles. This might affect the interpretation of our findings.

In conclusion, we failed to find evidence supporting the previous findings on the association between SSB consumption and metabolic syndrome. Prospective studies are needed to further explore for this association.

\section{Acknowledgements}

This program was conducted by Isfahan Cardiovascular Research Center (ICRC) (a WHO Collaborating Center) in collaboration with Isfahan Provincial Health Office, both affiliated to Isfahan University of Medical Sciences (IUMS). The program was supported by grant No. 31309304 of the Iranian Budget and Planning Organization, as well as the Deputy for Health of the Iranian Ministry of Health, Treatment and Medical Education, and the Iranian Heart Foundation (IHF). We are thankful to the collaborating teams in ICRC, Isfahan Provincial Health Office, Najaf-Abad Health Office and Arak University of Medical Sciences.

\section{Disclosure Statement}

The authors declared no conflicts of interest.

\section{References}

1 Resnick H, Jones K, Ruotolo G, Jain A, Henderson J, Lu W, Howard B: Insulin resistance, the metabolic syndrome, and risk of incident cardiovascular disease in nondiabetic American Indians. Diabetes Care 2003;26:861-867.

2 McNeill A, Rosamond W, Girman C, Golden S, Schmidt M, East H, Ballantyne C, Heiss G: The metabolic syndrome and 11-year risk of incident cardiovascular disease in the atherosclerosis risk in communities study. Diabetes Care 2005;28:385-390.

3 Lakka H, Laaksonen D, Lakka T, Niskanen L, Kumpusalo E, Tuomilehto J, Salonen J: The metabolic syndrome and total and cardiovascular disease mortality in middle-aged men. JAMA 2002;288:2709-2716.

4 Antonopoulos S: Third report of the National Cholesterol Education Program (NCEP) expert panel on detection, evaluation, and treatment of high blood cholesterol in adults (Adult Treatment Panel III) final report. Circulation 2002;106:3143-3421.

5 Reaven P: Metabolic syndrome. J Insur Med 2004;36:132-142.

6 Ford E, Giles W, Dietz W: Prevalence of the metabolic syndrome among US adults: findings from the third National Health and Nutrition Examination Survey. JAMA 2002;287:356-359. 
Khosravi-Boroujeni et al.: Consumption of Sugar-Sweetened Beverages in Relation to the Metabolic Syndrome among Iranian Adults

7 Azizi F, Etemadi A, Salehi P, Zahedi S: Prevalence of metabolic syndrome in an urban population: Tehran Lipid and Glucose Study. TUMJ 2003;61:389-399

- 8 Esmaillzadeh A, Mirmiran P, Azadbakht L, Etemadi A, Azizi F: High prevalence of the metabolic syndrome in Iranian adolescents. Obesity 2006;14:377-382.

- 9 Esmaillzadeh A, Kimiagar M, Mehrabi Y, Azadbakht L, Hu F, Willett W: Fruit and vegetable intakes, C-reactive protein, and the metabolic syndrome. Am J Clin Nutr 2006;84:1489-1497.

10 Azadbakht L, Esmaillzadeh A: Red meat intake is associated with metabolic syndrome and the plasma c-reactive protein concentration in women. J Nutr 2009;139:335-339.

11 Oh SW, Yoon YS, Lee ES, Kim WK, Park C, Lee S, Jeong EK, Yoo T: Association between cigarette smoking and metabolic syndrome. Diabetes Care 2005;28:2064-2066.

$\$ 12$ Miyatake N, Nishikawa H, Morishita A, Kunitomi M, Wada J, Suzuki H, Takahashi K, Makino H, Kira S, Fujii M: Daily walking reduces visceral adipose tissue areas and improves insulin resistance in japanese obese subjects. Diabetes Res Clin Pract 2002;58:101-107.

13 Lutsey P, Steffen L, Stevens J: Dietary intake and the development of the metabolic syndrome: The Atherosclerosis Risk in Communities Study. Circulation 2008;117:754-761.

$\checkmark 14$ Azadbakht L, Mirmiran P, Esmaillzadeh A, Azizi F: Dairy consumption is inversely associated with the prevalence of the metabolic syndrome in Tehranian adults. Am J Clin Nutr 2005;82:523-530.

15 Esmaillzadeh A, Mirmiran P, Azizi F: Whole-grain consumption and the metabolic syndrome: a favorable association in Tehranian adults. Eur J Clin Nutr 2004;59:353-362.

16 Azadbakht L, Surkan PJ, Esmaillzadeh A, Willett WC: The dietary approaches to stop hypertension eating plan affects C-reactive protein, coagulation abnormalities, and hepatic function tests among type 2 diabetic patients. J Nutr 2011;141:1083-1088.

17 Nielsen S, Popkin B: Changes in beverage intake between 1977 and 2001. Am J Prev Med 2004;27:205-210.

18 Vereecken C, Inchley J, Subramanian S, Hublet A, Maes L: The relative influence of individual and contextual socio-economic status on consumption of fruit and soft drinks among adolescents in Europe. Eur J Pub Health 2005;15:224-232.

19 Hejazi N, Mazloom Z: Socioeconomic status, youth's eating patterns and meals consumed away from home. Pakistan J Biol Sci 2009;12:730-733.

20 Karimi-Shahanjarini A, Omidvar N, Bazargan M, Rashidian A, Majdzadeh R, Shojaeizadeh D: Iranian female adolescent's views on unhealthy snacks consumption: a qualitative study. Iranian J Publ Health 2010;39: 92-101

21 James J, Thomas P, Cavan D, Kerr D: Preventing childhood obesity by reducing consumption of carbonated drinks: cluster randomised controlled trial. BMJ 2004;328:1237-1239.

-22 Schulze M, Manson J, Ludwig D, Colditz G, Stampfer M, Willett W, Hu F: Sugar-sweetened beverages, weight gain, and incidence of type 2 diabetes in young and middle-aged women. JAMA 2004;292:927-934.

23 Parks E, Hellerstein M: Carbohydrate-induced hypertriacylglycerolemia: Historical perspective and review of biological mechanisms1. Am J Clin Nutr 2000;71:412-433.

24 Drewnowski A, Bellisle F: Liquid calories, sugar, and body weight. Am J Clin Nutr 2007;85:651-661.

25 Dhingra R, Sullivan L, Jacques P, Wang T, Fox C, Meigs J, D’Agostino R, Gaziano J, Vasan R: Soft drink consumption and risk of developing cardiometabolic risk factors and the metabolic syndrome in middleaged adults in the community. Circulation 2007;116:480-488.

26 Pereira MA, Jacobs Jr DR, Van Horn L, Slattery ML, Kartashov AI, Ludwig DS: Dairy consumption, obesity, and the insulin resistance syndrome in young adults: the Cardia Study. JAMA 2002;287:2081-2089.

-27 Sarrafzadegan N, Kelishadi R, Baghaei A, Hussein Sadri G, Malekafzali H, Mohammadifard N, Rabiei K, Bahonar A, Sadeghi M, O'Laughlin J: Metabolic syndrome: an emerging public health problem in Iranian women: Isfahan Healthy Heart Program. Int J Cardiology 2008;131:90-96.

28 Sarraf-Zadegan N, Malek Afzali H, Baghaei M, Mohamadi Fard N, Shahrokhi S: Isfahan Healthy Heart Program: a comprehensive integrated community-based program for cardiovascular disease prevention and control. Acta Cardiol 2003;58:309-320.

-29 Sarrafzadegan N, Baghaei A, Sadri G, Kelishadi R: Isfahan Healthy Heart Program: evaluation of comprehensive, community-based interventions for non-communicable disease prevention. Prevent Contr 2006; 2:73-84.

-30 Friedewald WT, Levy RI, Fredrickson DS: Estimation of the concentration of low-density lipoprotein cholesterol in plasma, without use of the preparative ultracentrifuge. Clin Chem 1972;18:499-502.

31 Pereira M, FitzerGerald S, Gregg E, Joswiak M, Ryan W, Suminski R, Utter A, Zmuda J: A collection of physical activity questionnaires for health-related research. Med Sci Sports Exerc 1997;29(6 suppl):S1-205.

-32 Fung TT, Malik V, Rexrode KM, Manson JAE, Willett WC, Hu FB: Sweetened beverage consumption and risk of coronary heart disease in women. Am J Clin Nutr 2009;89:1037-1042.

33 Nettleton JA, Lutsey PL, Wang Y, Lima JA, Michos ED, Jacobs DR: Diet soda intake and risk of incident metabolic syndrome and type 2 diabetes in the Multi-Ethnic Study of Atherosclerosis (MESA). Diabetes Care 2009;32:688-694.

34 Duffey KJ, Gordon-Larsen P, Steffen LM, Jacobs DR, Popkin BM: Drinking caloric beverages increases the risk of adverse cardiometabolic outcomes in the Coronary Artery Risk Development in Young Adults (CARDIA) Study. Am J Clin Nutr 2010;92:954-959. 
Khosravi-Boroujeni et al.: Consumption of Sugar-Sweetened Beverages in Relation to the Metabolic Syndrome among Iranian Adults

-35 Palmer JR, Boggs DA, Krishnan S, Hu FB, Singer M, Rosenberg L: Sugar-sweetened beverages and incidence of type 2 diabetes mellitus in African American women. Arch Intern Med 2008;168:1487-1492.

-36 Forshee RA, Anderson PA, Storey ML: Sugar-sweetened beverages and body mass index in children and adolescents: a meta-analysis. Am J Clin Nutr 2008;87:1662-1671.

37 Bachman CM, Baranowski T, Nicklas TA: Is there an association between sweetened beverages and adiposity? Nutr Rev 2006;64:153-174.

-38 Forshee RA, Storey ML, Allison DB, Glinsmann WH, Hein GL, Lineback DR, Miller SA, Nicklas TA, Weaver GA White JS: A critical examination of the evidence relating high fructose corn syrup and weight gain. Crit Rev Food Sci Nutr 2007;47:561-582.

39 Pereira MA: The possible role of sugar-sweetened beverages in obesity etiology: a review of the evidence. Int J Obes 2006;30:S28-S36.

40 DiMeglio DP, Mattes RD: Liquid versus solid carbohydrate: effects on food intake and body weight. Int J Obes 2000;24:794-800.

41 Ebbeling CB, Feldman HA, Osganian SK, Chomitz VR, Ellenbogen SJ, Ludwig DS: Effects of decreasing sugarsweetened beverage consumption on body weight in adolescents: a randomized, controlled pilot study. Pediatrics 2006;117:673-680.

-42 Bray GA, Nielsen SJ, Popkin BM: Consumption of high-fructose corn syrup in beverages may play a role in the epidemic of obesity. Am J Clin Nutr 2004;79:537-543.

43 Forshee RA, Anderson PA, Storey ML: The role of beverage consumption, physical activity, sedentary behavior, and demographics on body mass index of adolescents. Int J Food Sci Nutr 2004;55:463-478.

44 Forshee RA, Storey ML: Total beverage consumption and beverage choices among children and adolescents. Int J Food Sci Nutr 2003;54:297-307.

45 Hein GL, Storey ML, White JS, Lineback DR: Highs and lows of high fructose corn syrup: a report from the Center for Food and Nutrition Policy and its Ceres workshop. Nutr Today 2005;40:253-256.

-46 Paynter NP, Yeh HC, Voutilainen S, Schmidt MI, Heiss G, Folsom AR, Brancati FL, Kao WH: Coffee and sweetened beverage consumption and the risk of type 2 diabetes mellitus. Am J Epidemiol 2006;164:10751084.

47 Yoo S, Nicklas T, Baranowski T, Zakeri IF, Yang SJ, Srinivasan SR, Berenson GS: Comparison of dietary intakes associated with metabolic syndrome risk factors in young adults: The Bogalusa Heart Study. Am J Clin Nutr 2004;80:841-848.

48 Sturt J: Higher consumption of sugar-sweetened beverages is associated with increased risk of developing type 2 diabetes or metabolic syndrome. Evid Based Nurs 2011;14:35-39.

49 Malik VS, Schulze MB, Hu FB: Intake of sugar-sweetened beverages and weight gain: a systematic review. Am J Clin Nutr 2006;84:274-288.

50 Hu FB, Malik VS: Sugar-sweetened beverages and risk of obesity and type 2 diabetes: epidemiologic evidence. Physiol Behav 2010;100:47-54.

51 Ko G, So W, Chow C, Wong P, Tong S, Hui S, Kwok R, Chan A, Chan C, Chan J: Risk associations of obesity with sugar-sweetened beverages and lifestyle factors in chinese: THE 'Better Health for Better Hong Kong' Health Promotion Campaign. Eur J Clin Nutr 2010;64:1386-1392.

52 Malik VS, Hu FB: Sugar-sweetened beverages and health: where does the evidence stand? Am J Clin Nut 2011;94:1161-1162.

53 Malik VS, Popkin BM, Bray GA, Després JP, Willett WC, Hu FB: Sugar-sweetened beverages and risk of metabolic syndrome and type 2 diabetes. Diabetes Care 2010;33:2477-2483.

54 De Koning L, Malik VS, Rimm EB, Willett WC, Hu FB: Sugar-sweetened and artificially sweetened beverage consumption and risk of type 2 diabetes in men. Am J Clin Nutr 2011;93:1321-1327.

55 Frost G, Leeds AA, Dore CJ, Madeiros S, Brading S, Dornhorst A: Glycaemic index as a determinant of serum HDL-cholesterol concentration. Lancet 1999;353:1045-1048.

56 Willett W, Manson JA, Liu S: Glycemic index, glycemic load, and risk of type 2 diabetes. Am J Clin Nutr 2002; 76:274S-280s.

57 Janssens JP, Shapira N, Debeuf P, Michiels L, Putman R, Bruckers L, Renard D, Molenberghs G: Effects of soft drink and table beer consumption on insulin response in normal teenagers and carbohydrate drink in youngsters. Eur J Cancer Prev 1999;8:289-295.

-58 McKeown NM, Meigs JB, Liu S, Saltzman E, Wilson PWF, Jacques PF: Carbohydrate nutrition, insulin resistance, and the prevalence of the metabolic syndrome in the Framingham Offspring Cohort. Diabetes Care 2004;27:538-546. 